\title{
Invariants for Weighted Digraphs under One-Sided State Splittings
}

\author{
Sheng Chen, Xiaomei Chen, and Chao Xia \\ Department of Mathematics, Harbin Institute of Technology, Harbin 150001, China \\ Correspondence should be addressed to Sheng Chen; schenhit@gmail.com
}

Received 7 February 2013; Accepted 9 April 2013

Academic Editor: Md Sazzad Chowdhury

Copyright (c) 2013 Sheng Chen et al. This is an open access article distributed under the Creative Commons Attribution License, which permits unrestricted use, distribution, and reproduction in any medium, provided the original work is properly cited.

Using Matrix-Forest theorem and Matrix-Tree theorem, we present some invariants for weighted digraphs under state in-splittings or out-splittings.

\section{Introduction}

State in-splittings and out-splittings are very important operations in the theory of one-sided, or two-sided Markov shifts $([1,2])$. Lind and Tuncel introduced a spanning tree invariant for Markov shifts in [3]. Spanning tree invariants are further studied in [4-6]. Motivated by these works, we consider some other graph structures like cycles and forests and present some invariants for weighted digraphs under state insplittings or out-splittings.

Firstly we give some basic definitions in graph theory and a brief introduction of Matrix-Forest theorem for digraphs. Readers can refer to $[7,8]$ for more details.

In this paper, a digraph is an ordered pair $D=(V, E)$ of finite sets, where $V$ is called the vertex set and $E \subseteq V \times V$ is called the edge set. For an edge $(u, v) \in E, u$ and $v$ are called the initial and terminal ends of the edge, respectively. The number of edges having $u$ as the initial end is defined to be the outdegree of $u$ and denoted by $d(u)$. The number of edges having $v$ as the terminal end is defined to be the indegree of $v$. A walk of length $n$ is a sequence of edges $\left\{\left(u_{i}, u_{i+1}\right)\right\}(i=$ $1, \ldots, n)$ and can be denoted by $\left(u_{1}, u_{2}, \ldots, u_{n+1}\right)$; moreover, if $u_{n+1}$ is the same as $u_{1}$, we call the walk a closed one. A directed forest is a digraph without closed walks such that the indegree of each vertex is no more than one. The vertices with indegree zero of a forest are called roots. We say that $D_{0}=\left(V_{0}, E_{0}\right)$ is a spanning subgraph of $D$ if $V_{0}=V$ and $E_{0} \subseteq E$.

Suppose that $D$ is a digraph with vertex set $V(D)=\{1$, $\ldots, n\}$. Let $w: E(D) \rightarrow \mathbb{R}^{+}$be a weight function on the edge set. We then say that $\mathscr{D}=(D, w)$ is a weighted digraph and $M=(w(i, j))_{n \times n}$ is the weight matrix of $\mathscr{D}$. The Kirchhoff matrix of $\mathscr{D}$ is defined as $L=R-M$, where $R=\left(r_{i, j}\right)$ is a diagonal matrix and $r_{i, i}=\sum_{j=1}^{n} w(i, j)$. The product of the weights of all edges that belong to a subgraph $\mathscr{H}$ of $\mathscr{D}$ is defined to be the weight of $\mathscr{H}$ and denoted by $w(\mathscr{H})$.

Let $\mathscr{F}(\mathscr{D})=\mathscr{F}$ be the set of all spanning rooted forests of $\mathscr{D}$ and $\mathscr{F}^{i \rightarrow j}(\mathscr{D})=\mathscr{F}^{i \rightarrow j}$ the set of those spanning rooted forests of $\mathscr{D}$ such that $i$ and $j$ belong to the same tree rooted at $i$. For a matrix $A, A^{i, j}$ denotes the cofactor of the $(i, j)$-entry of $A$. The Matrix-Forest theorem then states as follows.

Lemma 1 (cf. [8]). Let $\mathscr{D}=(D, w)$ be a weighted digraph. Let $L$ be the Kirchhoff matrix of $\mathscr{D}$. Then one has

(1) $\sum_{F \in \mathscr{F}} w(F)=\operatorname{det}(I+L)$;

(2) for any $i, j \in V(D), \sum_{F \in \mathscr{F}^{i \rightarrow j}} w(F)=(I+L)^{i, j}$.

\section{Invariants for Weighted Digraphs under State In-Splitting}

Before giving the main result, we recall the definition of state in-splitting.

Definition 2. Let $\mathscr{D}=(D, w)$ be a weighted digraph. For a vertex $u$ of $D, E^{u}$ denotes the set of edges of $D$ with terminal end $u$. The state in-splitting of $\mathscr{D}$ at $u$ induces a new weighted digraph $\widetilde{\mathscr{D}}=(\widetilde{D}, \widetilde{w})$ in the following way: let $\mathcal{S}=$ 
$\left\{S_{1}, S_{2}, \ldots, S_{r}\right\}$ be a partition of $E^{u}$. The vertex set of the new digraph is $V(\widetilde{D})=(V(D) \backslash\{u\}) \bigcup\left\{u_{1}, u_{2}, \ldots, u_{r}\right\}$. The edge set $E(\widetilde{D})$ and weight $\widetilde{w}$ of $\widetilde{D}$ are defined as follows.

(i) For $x, y \in V(D) \backslash\{u\},(x, y) \in E(\widetilde{D})$ if and only if $(x, y) \in E(D)$ and in this case $\widetilde{w}(x, y)=w(x, y)$.

(ii) For $x \in V(D) \backslash\{u\},\left(x, u_{i}\right) \in E(\widetilde{D})$ if and only if $(x, u) \in$ $S_{i}$ and in this case $\widetilde{w}\left(x, u_{i}\right)=w(x, u)$.

(iii) For $x \in V(D) \backslash\{u\},\left(u_{i}, x\right) \in E(\widetilde{D})$ if and only if $(u, x) \epsilon$ $E(D)$ and in this case $\widetilde{w}\left(u_{i}, x\right)=w(u, x)$.

(iv) If $(u, u) \in S_{i}$, then $\left(u_{j}, u_{i}\right) \in E(\widetilde{D})$, for $j=1,2, \ldots, r$, and in this case $\widetilde{w}\left(u_{j}, u_{i}\right)=w(u, u)$.

For more details about state splittings, readers can refer to $[2,3,9]$. Now we give the definition of our new invariant.

Definition 3. Let $\mathscr{D}=(D, w)$ be a weighted digraph. We define $W_{k}(\mathscr{D})(k \geq 1)$ as

$$
W_{k}(\mathscr{D})=\sum_{v} d(v) \sum_{C \in C_{v}^{k}} w(C),
$$

where $v$ runs over $V(D)$ and $C_{v}^{k}$ denotes the set of closed walks of $\mathscr{D}$ with length $k$ at vertex $v$. Furthermore, we define the generating function $W_{\mathscr{D}}(t)$ as

$$
W_{\mathscr{D}}(t)=\sum_{k \geq 1} W_{k}(\mathscr{D}) t^{k} .
$$

Let $A$ be a square matrix. The trace of $A$ is defined to be the sum of the elements on the main diagonal and denoted by $\operatorname{tr}(A)$. For a digraph $D$, the diagonal matrix $O(D)=\left(o_{i, i}\right)$ denotes the outdegree matrix of $D$ that is, $o_{i, i}=d\left(v_{i}\right)$. Then we have the following result.

Theorem 4. Let $\mathscr{D}$ be a weighted digraph with weight matrix $M$. Then $W_{\mathscr{D}}(t)$ is an invariant under state in-splitting and can be computed in the following way:

$$
W_{\mathscr{D}}(t)=\frac{\operatorname{tr}(O \cdot \operatorname{adj}(I-t M))}{\operatorname{det}(I-t M)}-\operatorname{tr}(O) .
$$

Proof. We firstly prove the invariance of $W_{k}(\mathscr{D})$ for $k \geq 1$. Without loss of generality, if there is a loop at vertex $u$, we assume that it belongs to $S_{1}$, where $\mathcal{S}=\left\{S_{1}, S_{2}, \ldots, S_{r}\right\}$ denotes the partition of $E^{u}$ as in the definition of state insplitting.

We define the mapping

$$
\varphi: \bigcup_{v \in V(D)} C_{v}^{k}(\mathscr{D}) \longrightarrow \bigcup_{v \in V(\widetilde{D})} C_{v}^{k}(\widetilde{D})
$$

in the following way: for a closed walk $C$ of $\mathscr{D}$ with length $k$, if $C=(u, u, \ldots, u)$, then $\varphi(C)=\left(u_{1}, u_{1}, \ldots, u_{1}\right)$; otherwise, we replace each maximum path of $C$ of the form $(v, u, u, \ldots, u)(v \neq u)$ with $\left(v, u_{i}, u_{1}, \ldots, u_{1}\right)$ if $(v, u) \in S_{i}$. it is not difficult to see that

$$
\varphi: C_{v}^{k}(\mathscr{D}) \longrightarrow C_{v}^{k}(\widetilde{D}),
$$
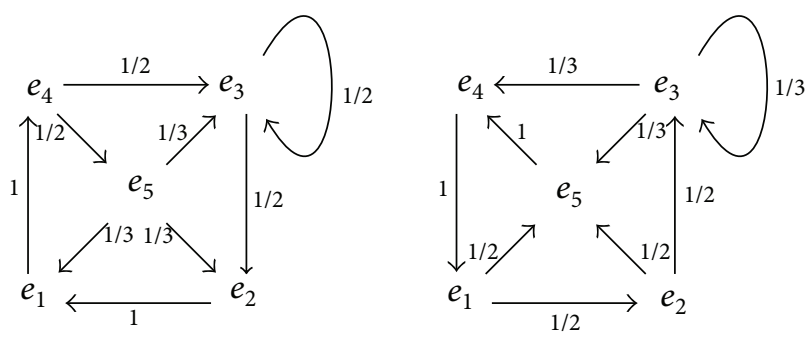

Figure 1

where $v \neq u$, and

$$
\varphi: C_{u}^{k}(\mathscr{D}) \longrightarrow \bigcup_{i=1}^{r} C_{u_{i}}^{k}(\widetilde{D})
$$

are both weight-preserving bijections.

Since $d(v)(v \neq u)$ is the same for $\mathscr{D}$ and $\widetilde{D}$ and $d(u)=$ $d\left(u_{1}\right)=d\left(u_{2}\right)=\cdots=d\left(u_{r}\right)$, we know that $W_{k}(\mathscr{D})=W_{k}(\widetilde{D})$ for $k \geq 1$, and the invariance of $W_{\mathscr{D}}(t)$ follows.

Finally, we notice that $W_{k}(\mathscr{D})=\operatorname{tr}\left(O M^{k}\right)$. Thus

$$
\begin{aligned}
W_{\mathscr{D}}(t) & =\sum_{k \geq 1} \operatorname{tr}\left\{O \cdot(t M)^{k}\right\} \\
& =\operatorname{tr}\left\{O \cdot \sum_{k \geq 1}(t M)^{k}\right\} \\
& =\frac{\operatorname{tr}(O \cdot \operatorname{adj}(I-t M))}{\operatorname{det}(I-t M)}-\operatorname{tr}(O) .
\end{aligned}
$$

Example 5. Let $\mathscr{D}=(D, w)$ be a weighted digraph as in the left of Figure 1. $\widehat{D}$ is the opposite of $D$ (see the right of Figure 1), that is, the digraph obtained from $D$ by reversing the direction of all its edges. It is easy to see that $D$ and $\widehat{D}$ have the same outdegree sequence $\{1,1,2,2,3\}$. The weight of any edge $(u, v) \in E(D)$ or $(u, v) \in E(\widehat{D})$ is defined to be $1 / d(u)$. Since $W_{3}(\mathscr{D})=5 / 4$ and $W_{3}(\widehat{D})=19 / 9$, we know that $\widehat{\mathscr{D}}$ cannot be obtained from $\mathscr{D}$ by a sequence of in-splittings or reverse operations.

Let $P$ be a nonnegative matrix. $P$ is called row stochastic if the summation of each row equals 1 and column stochastic if the summation of each column equals $1 P$ is called double stochastic if it is row and column stochastic.

Definition 6. Let $P$ be a row-stochastic matrix and $t$ a real positive number. Let $\mathscr{D}$ be the weighted digraph with weight matrix $M=t P$. We define $K(\mathscr{D}, t)$ as

$$
K(\mathscr{D}, t)=(1+t) \frac{\sum_{v} d(v) \sum_{F_{v}} w\left(F_{v}\right)}{\sum_{F} w(F)},
$$

where $v$ runs over all vertices of $V(D), F$ runs over all spanning directed forests of $D$, and $F_{v}$ runs over all spanning directed forests including $v$ as a root. 
In general, $K(\mathscr{D}, t)$ is not an invariant under state insplitting, but the following result shows that it indeed reflects some invariance.

Corollary 7. Let $P$ be a row-stochastic matrix and t a real positive number. Let $\mathscr{D}$ be a weighted digraph with weight matrix $M=t P$. Then $K(\mathscr{D}, t)-K(\widetilde{\mathscr{D}}, t)$ is an integer independent of $t$.

Proof. Let $O=\left(o_{i, j}\right)$ be the outdegree matrix of $D$. Then we get by Lemma 1 that

$$
\begin{aligned}
K(\mathscr{D}, t) & =(1+t) \frac{\sum_{v} d(v) \sum_{F_{v}} w\left(F_{v}\right)}{\sum_{F} w(F)} \\
& =(1+t) \frac{\operatorname{tr}(O \cdot \operatorname{adj}[I+(t I-M)])}{\operatorname{det}[I+(t I-M)]} \\
& =\operatorname{tr}\left(O \cdot\left[I-\frac{t}{1+t} P\right]^{-1}\right) .
\end{aligned}
$$

Since $P$ is stochastic and $1 /(1+t) \in(0,1)$, we have

$$
\left[I-\frac{t}{1+t} P\right]^{-1}=[I-r(t P)]^{-1}=\sum_{i \geq 0}(M)^{i} r^{i}
$$

where $r=1 /(1+t)$.

Therefore

$$
\begin{aligned}
K(\mathscr{D}, t) & =\operatorname{tr}\left(O \cdot\left[I-\frac{t}{1+t} P\right]^{-1}\right) \\
& =\sum_{i \geq 0} \operatorname{tr}\left\{O(M)^{i}\right\} r^{i} \\
& =W_{\mathscr{D}}(r)+\operatorname{tr}(O) .
\end{aligned}
$$

By Theorem 4, we know that $W_{\mathscr{D}}(r)$ is an invariant under in-splitting; thus

$$
K(\mathscr{D}, t)-K(\widetilde{\mathscr{D}}, t)=\operatorname{tr}(O)-\operatorname{tr}(\widetilde{O}) \in \mathbb{Z} .
$$

The result follows.

Lind and Tuncel defined a spanning tree invariant $\tau(\mathscr{D})$ for Markov shifts in [3] as follows:

$$
\tau(\mathscr{D})=\sum_{T} w(T) .
$$

Here the weight matrix $P$ of $\mathscr{D}$ is an irreducible row-stochastic matrix, and $T$ runs over all spanning trees of $\mathscr{D}$.

By considering the outdegree matrix as in Definitions 3 and 6 , we can define a new spanning tree invariant as

$$
\tau_{d}(\mathscr{D})=\sum_{T} d(T) w(T),
$$

where $T$ is as above, and $d(T)$ denotes the outdegree of the root of $T$.

Corollary 8. $\tau_{d}(\mathscr{D})$ is an invariant under in-splitting.
Proof. Let $P$ be the weight matrix of $\mathscr{D}$ and thus row stochastic as in [3]. By the Matrix-Tree theorem (Theorem 2 in $[8]$ ), we have

$$
\begin{aligned}
\tau_{d}(\mathscr{D}) & =\operatorname{tr}(O \cdot \operatorname{adj}[I-P]) \\
& =\lim _{t \rightarrow 1^{-}}\left\{\operatorname{det}[I-t P] \cdot \operatorname{tr}\left(O \cdot[I-t P]^{-1}\right)\right\} \\
& =\lim _{t \rightarrow 1^{-}}\left\{\operatorname{det}[I-t P] \cdot \sum_{i \geq 0} \operatorname{tr}\left(O P^{i}\right) t^{i}\right\} \\
& =\lim _{t \rightarrow 1^{-}}\left\{\operatorname{det}[I-t P] \cdot\left(W_{\mathscr{D}}(t)+\operatorname{tr}(O)\right)\right\} .
\end{aligned}
$$

By Theorem 4 , we know that $W_{\mathscr{D}}(t)$ is an invariant under state in-splitting. it is also well known that $\operatorname{det}[I-t P]$ is an invariant under state splitting. Therefore

$$
\tau_{d}(\mathscr{D})-\tau_{d}(\widetilde{D})=\lim _{t \rightarrow 1^{-}}\{\operatorname{det}[I-t P] \cdot(\operatorname{tr}(O)-\operatorname{tr}(\widetilde{O}))\} .
$$

Since $\operatorname{tr}(O)-\operatorname{tr}(\widetilde{O})$ is a constant and $\lim _{t \rightarrow 1^{-}} \operatorname{det}[I-t P]=0$, we have

$$
\tau_{d}(\mathscr{D})-\tau_{d}(\widetilde{\mathscr{D}})=0 .
$$

The result follows.

Let $\mathscr{D}=(D, w)$ be a weighted digraph. The outweighted line digraph $L^{+}(\mathscr{D})=\left(L(D), w^{+}\right)$of $\mathscr{D}$ is a weighted digraph defined in the following way: the vertex set of $L(D)$ is $E(D) ;((u, v),(x, y)) \in E(L(D))$ if and only if $v=x$, and in this case, $w^{+}(((u, v),(x, y)))=w(x, y)$. Similarly, if we let $w^{-}(((u, v),(x, y)))=w(u, v)$ in the above definition, then we get the in-weighted line digraph $L^{-}(\mathscr{D})=$ $\left(L(D), w^{-}\right)$. Galeana-Sánchez and Gómez show that $L^{+}(\mathscr{D})$ can be obtained by sequences of state in-splittings from $\mathscr{D}$ (see Proposition 2.2 in [9], which has a small typo there by stating $L^{-}(\mathscr{D})$ can be obtained by sequences of state insplittings). Now the following conclusion is an immediate result of Corollary 8.

Corollary 9. $\tau_{d}(\mathscr{D})$ is an invariant under out-weighted line digraph operation.

\section{The State Out-Splitting Case}

Let $P$ be a row-stochastic matrix. Let $\mathscr{D}=(D, W)$ be the weighted digraph with weight matrix $W=P$. We first give the definition of state out-splitting, which is a little more complicated than the case of state in-splitting. Readers can refer to [3] for more details.

Definition 10. For a vertex $u$ of $D$, let $E^{* u}$ denote the set of edges of $D$ with initial end $u$. The state out-splitting of $\mathscr{D}$ at $u$ induces a new weighted digraph $\widetilde{D}^{*}=\left(\widetilde{D}^{*}, \widetilde{w}\right)$ in the following way: let $\mathcal{S}^{*}=\left\{S_{1}^{*}, S_{2}^{*}, \ldots, S_{r}^{*}\right\}$ be a partition of $E^{* u}$. Let $q_{i}$ denote the sum of the weights of edges in $S_{i}^{*}$. The vertex set of the new digraph is $V\left(\widetilde{D}^{*}\right)=(V(D) \backslash$ $\{u\}) \bigcup\left\{u_{1}, u_{2} \ldots, u_{r}\right\}$. The edge set and weight of $\widetilde{D}^{*}$ are defined as follows. 
(i) For $x, y \in V(D) \backslash\{u\},(x, y) \in E\left(\widetilde{D}^{*}\right)$ if and only if $(x, y) \in E(D)$ and in this case $\widetilde{w}(x, y)=w(x, y)$.

(ii) For $y \in V(D) \backslash\{u\},\left(u_{i}, y\right) \in E\left(\widetilde{D}^{*}\right)$ if and only if $(u, y) \in S_{i}^{*}$ and in this case $\widetilde{w}\left(u_{i}, y\right)=w(u, y) / q_{i}$.

(iii) For $x \in V(D) \backslash\{u\},\left(x, u_{i}\right) \in E\left(\widetilde{D}^{*}\right)$ if and only if $(x, u) \in E(D)$ and in this case $\widetilde{w}\left(u_{i}, x\right)=q_{i} w(x, u)$.

(iv) If $(u, u) \in S_{i}^{*}$, then $\left(u_{i}, u_{j}\right) \in E\left(\widetilde{D}^{*}\right)$, for $j=1,2, \ldots, r$, and in this case $\widetilde{w}\left(u_{i}, u_{j}\right)=w(u, u) q_{j} / q_{i}$.

In the definition of $W_{k}(\mathscr{D})$ and $W_{D}(t)$, by replacing outdegrees with indegrees, we get $W_{k}^{*}(\mathscr{D})$ and $W_{\mathscr{D}}^{*}(t)$; that is,

$$
\begin{gathered}
W_{k}^{*}(\mathscr{D})=\sum_{v} d^{*}(v) \sum_{C \in C_{v}^{k}} w(C), \\
W_{\mathscr{D}}^{*}(t)=\sum_{k \geq 1} W_{k}^{*}(\mathscr{D}) t^{k},
\end{gathered}
$$

where $d^{*}(v)$ is the indegree of $v$.

Theorem 11. Let $P$ be a row-stochastic matrix. Let $\mathscr{D}$ be the weighted digraph with weight matrix $P$. Then $W_{D}^{*}(t)$ is an invariant under state out-splitting, and can be computed as

$$
W_{\mathscr{D}}^{*}(t)=\frac{\operatorname{tr}\left(O^{*} \cdot \operatorname{adj}(I-t P)\right)}{\operatorname{det}(I-t P)}-\operatorname{tr}\left(O^{*}\right)
$$

where $O^{*}$ is the indegree matrix of $D$.

Proof. We just need to prove the invariance of $W_{k}^{*}(\mathscr{D})$ for $k \geq 1$. Without loss of generality, if there is a loop at vertex $u$, we assume that it belongs to $S_{1}^{*}$, where $\mathcal{S}^{*}=\left\{S_{1}^{*}, S_{2}^{*}, \ldots, S_{r}^{*}\right\}$ denotes the partition of $E^{* u}$ as in the definition of state outsplitting.

We define the mapping

$$
\varphi: \bigcup_{v \in V(D)} C_{v}^{k}(\mathscr{D}) \longrightarrow \bigcup_{v \in V(\widetilde{D})} C_{v}^{k}\left(\widetilde{\mathscr{D}}^{*}\right)
$$

in the following way: for a closed walk $C$ of $\mathscr{D}$ with length $k$, if $C=(u, u, \ldots, u)$, then $\varphi(C)=\left(u_{1}, u_{1}, \ldots, u_{1}\right)$; otherwise, we replace each maximum path of $C$ of the form $(u$, $u, \ldots, u, u, v)(v \neq u)$ with $\left(u_{1}, u_{1}, \ldots, u_{1}, u_{i}, v\right)$ if $(u, v) \in S_{i}^{*}$. By the definition of state out-splitting, it is not difficult to prove that

$$
\varphi: C_{v}^{k}(\mathscr{D}) \longrightarrow C_{v}^{k}\left(\widetilde{\mathscr{D}}^{*}\right)
$$

where $v \neq u$, and

$$
\varphi: C_{u}^{k}(\mathscr{D}) \longrightarrow \bigcup_{i=1}^{r} C_{u_{i}}^{k}\left(\widetilde{\mathscr{D}}^{*}\right)
$$

are both bijections.

We now prove that they are also weight-preserving. In fact, if $C=(u, u, \ldots, u)$, then $w(C)=w(\varphi(C))$, since $\widetilde{w}\left(u_{i}\right.$, $\left.u_{i}\right)=w(u, u)$. On the other hand, for any walk of $C$ of the form $S=(r, u, \ldots, u, u, v)(v \neq u)$, we have $w(S)=w(r$, $u) w(u, u)^{k} w(u, v)$.
(1) If $(u, v) \in S_{1}^{*}$, we have

$$
\begin{aligned}
w(\varphi(S)) & =w\left(r, u_{1}\right) w\left(u_{1}, u_{1}\right)^{k} w\left(u_{1}, v\right) \\
& =\frac{q_{1} w(r, u) w(u, u)^{k} w(u, v)}{q_{1}} \\
& =w(S) .
\end{aligned}
$$

(2) If $(u, v) \in S_{i}^{*}(i \neq 1)$ and $k \geq 1$, we have

$$
\begin{aligned}
w(\varphi(S)) & =w\left(r, u_{1}\right) w\left(u_{1}, u_{1}\right)^{k-1} w\left(u_{1}, u_{i}\right) w\left(u_{i}, v\right) \\
& =\frac{q_{1} w(r, u) w(u, u)^{k-1}\left(w(u, u) q_{i} / q_{1}\right) w(u, v)}{q_{i}} \\
& =w(S) .
\end{aligned}
$$

(3) If $(u, v) \in S_{i}^{*}(i \neq 1)$ and $k=0$, we have

$$
\begin{aligned}
w(\varphi(S)) & =w\left(r, u_{i}\right) w\left(u_{i}, v\right) \\
& =\frac{q_{i} w(r, u) w(u, v)}{q_{i}} \\
& =w(S) .
\end{aligned}
$$

Thus the maps above are weight preserving. Since $d^{*}(v)(v \neq u)$ is the same for $\mathscr{D}$ and $\widetilde{D}^{*}$, and $d^{*}(u)=d^{*}\left(u_{1}\right)=$ $d^{*}\left(u_{2}\right)=\cdots=d^{*}\left(u_{r}\right)$, we know that $W_{k}^{*}(\mathscr{D})=W_{k}^{*}\left(\widetilde{D}^{*}\right)$, for $k \geq 1$, and the invariance of $W_{\mathscr{D}}^{*}(t)$ follows.

The proof of the equality is similar to that of Theorem 4 .

Similarly, we can define $\tau_{d}^{*}(\mathscr{D})$ and prove that it is also an invariant under state out-splitting on the basis of the above result.

Now, we consider some weighted digraphs from [10] in the following two examples.

Example 12. The weight matrices of two weighted digraphs are as follows:

$$
A=\left[\begin{array}{ccc}
\frac{3}{8} & \frac{1}{2} & \frac{1}{8} \\
0 & \frac{4}{5} & \frac{1}{5} \\
\frac{2}{7} & \frac{4}{7} & \frac{1}{7}
\end{array}\right], \quad B=\left[\begin{array}{ccc}
\frac{1}{7} & 0 & \frac{6}{7} \\
\frac{5}{56} & \frac{3}{8} & \frac{15}{28} \\
\frac{2}{15} & \frac{1}{15} & \frac{4}{5}
\end{array}\right] .
$$

By some computation, we get that $W_{\mathscr{A}}(1 / 2)=1316 / 471$, $W_{\mathscr{B}}(1 / 2)=1615 / 471$, and $W_{\mathscr{A}}^{*}(1 / 2)=W_{\mathscr{B}}^{*}(1 / 2)=1559 / 471$. Thus $B$ cannot be archived by a sequence of in-splittings or reverse operations, but may be archived by a sequence of outsplittings or reverse operations. 
Example 13. The weight matrices of three weighted digraphs are as follows:

$$
A=\left[\begin{array}{ll}
\frac{1}{3} & \frac{2}{3} \\
\frac{1}{3} & \frac{2}{3}
\end{array}\right], \quad B=\left[\begin{array}{ll}
\frac{1}{3} & \frac{2}{3} \\
\frac{2}{3} & \frac{1}{3}
\end{array}\right], \quad C=\left[\begin{array}{ll}
\frac{2}{3} & \frac{1}{3} \\
\frac{1}{3} & \frac{2}{3}
\end{array}\right] .
$$

By some computation, we get that $\tau_{d}^{*}(A)=2=\tau_{d}(A)$, $\tau_{d}^{*}(B)=8 / 3=\tau_{d}(B), \tau_{d}^{*}(C)=4 / 3=\tau_{d}(C)$. Thus for any pair of them, we cannot get one from the other and by a sequence of in-splittings or reverse operations either nor by a sequence of out-splittings or reverse operations.

\section{Invariants for Weighted Digraphs with Double-Stochastic Matrices}

Let $\mathscr{D}=(D, P)$ be a weighted digraph. If the weight matrix $P$ is column stochastic, the weight distribution after state out-splitting can be defined in an easier way, that is, without multiplying by the coefficients about $q_{i}$ in Definition 10. Under this definition, we can get that $\tau_{d}^{*}(\mathscr{D})$ is still an invariant under state out-splitting, the proof of which is similar to that of Corollary 8. We also know from [9] that the in-weighted line digraph can be obtained by a sequence of such state out-splittings, so the following result is immediate.

Corollary 14. Let $\mathscr{D}=(D, P)$ be a weighted digraph. If the weight matrix $P$ is column stochastic, then $\tau_{d}^{*}(\mathscr{D})$ is an invariant under in-weighted line digraph operation.

Especially, if the weight matrix is doubly stochastic, we have the following result.

Corollary 15. Let $\mathscr{D}=(D, P)$ be a weighted digraph. If the weight matrix $P$ is doubly stochastic, then $\tau_{d}\left(L^{+}(\mathscr{D})\right)=$ $\tau_{d}^{*}\left(L^{-}(\mathscr{D})\right)$.

Proof. Since $P$ is doubly stochastic, we have by Corollary 8 that

$$
\tau_{d}\left(L^{+}(\mathscr{D})\right)=\tau_{d}(\mathscr{D})=\operatorname{tr}(O \cdot \operatorname{adj}[I-P])
$$

and by Corollary 14 that

$$
\tau_{d}^{*}\left(L^{-}(\mathscr{D})\right)=\tau_{d}^{*}(\mathscr{D})=\operatorname{tr}\left(O^{*} \cdot \operatorname{adj}[I-P]\right) .
$$

By Matrix-Tree theorem (Theorem 2 in [8]), we know that both $\operatorname{adj}[I-P]$ and $\operatorname{adj}\left[I-P^{\prime}\right]=(\operatorname{adj}[I-P])^{\prime}$ are rowconstant matrices, where $P^{\prime}$ is $P$ transposed. Thus adj $[I-P]$ is a constant matrix. Since the sum of indegrees is equal to that of outdegrees, the result follows.

\section{Acknowledgments}

The research was supported by National Natural Science Foundation of China (Grant nos. 11001064 and 11101105) and by the Scientific Research Foundation for the Returned Overseas Chinese Scholars, State Education Ministry. Xiaomei Chen was supported by China Scholarship Council.

\section{References}

[1] B. P. Kitchens, Symbolic Dynamics: One-Sided, Two-Sided and Countable State Markov Shifts, Springer, Berlin, Germany, 1998.

[2] D. Lind and B. Marcus, An Introduction to Symbolic Dynamics and Coding, Cambridge University Press, Cambridge, UK, 1995.

[3] D. Lind and S. Tuncel, "A spanning tree invariant for Markov shifts," in Codes, Systems, and Graphical Models, vol. 123 of The IMA Volumes in Mathematics and Its Applications, pp. 487-497, Springer, New York, NY, USA, 2001.

[4] R. B. Bapat, "On a conjecture concerning spanning tree invariants and loop systems," Linear Algebra and Its Applications, vol. 433, no. 8-10, pp. 1642-1645, 2010.

[5] R. Gómez, "On mean recurrence times of Markov chains and spanning tree invariants," Linear Algebra and Its Applications, vol. 433, no. 11-12, pp. 1714-1718, 2010.

[6] R. Gómez and J. M. Salazar, "Spanning tree invariants, loop systems and doubly stochastic matrices," Linear Algebra and Its Applications, vol. 432, no. 2-3, pp. 556-565, 2010.

[7] B. Bollobás, Modern Graph Theory, vol. 184, Springer, New York, NY, USA, 1998.

[8] P. Yu. Chebotarev and E. V. Shamis, "The Matrix-Forest theorem and measuring relations in small social groups," Automation and Remote Control, vol. 58, no. 9, pp. 1505-1514, 1997.

[9] H. Galeana-Sánchez and R. Gómez, "Monochromatic paths on edge colored digraphs and state splittings," Advances and Applications in Discrete Mathematics, vol. 4, no. 1, pp. 33-51, 2009.

[10] R. Cowen and E. M. Lungu, "When are two Markov chains the same?” Quaestiones Mathematicae, vol. 23, no. 4, pp. 507-513, 2000 . 


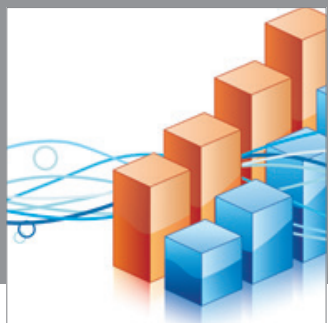

Advances in

Operations Research

mansans

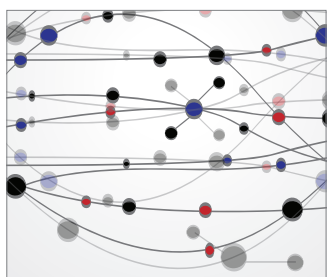

The Scientific World Journal
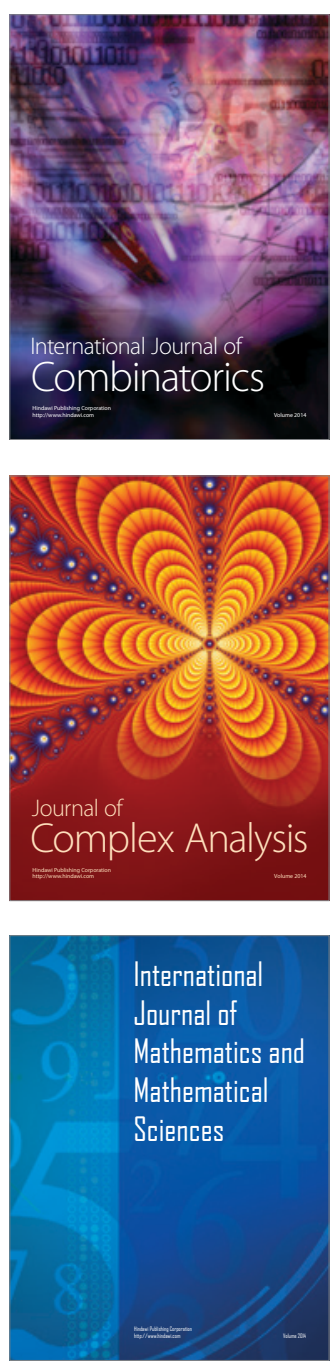
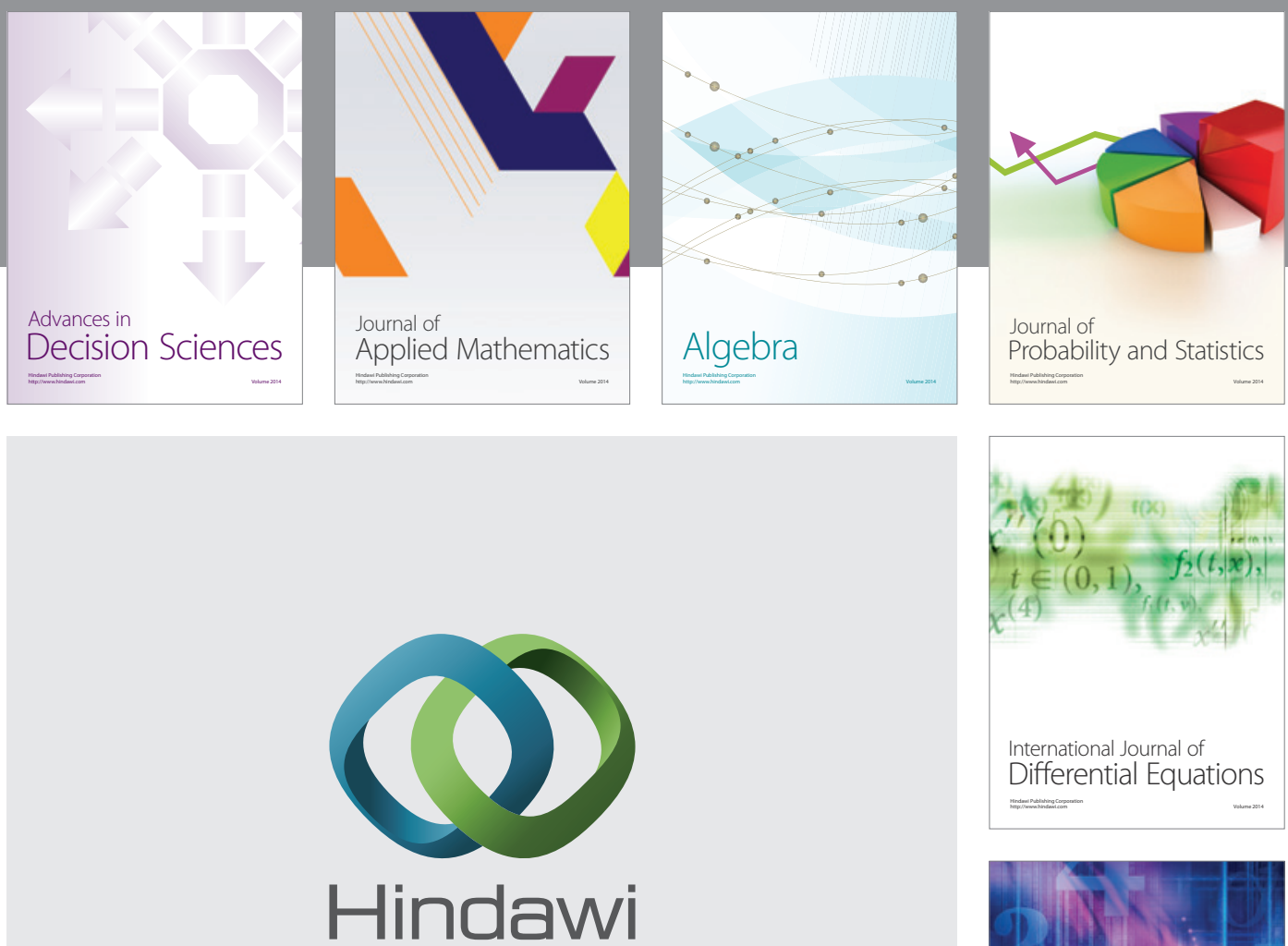

Submit your manuscripts at http://www.hindawi.com
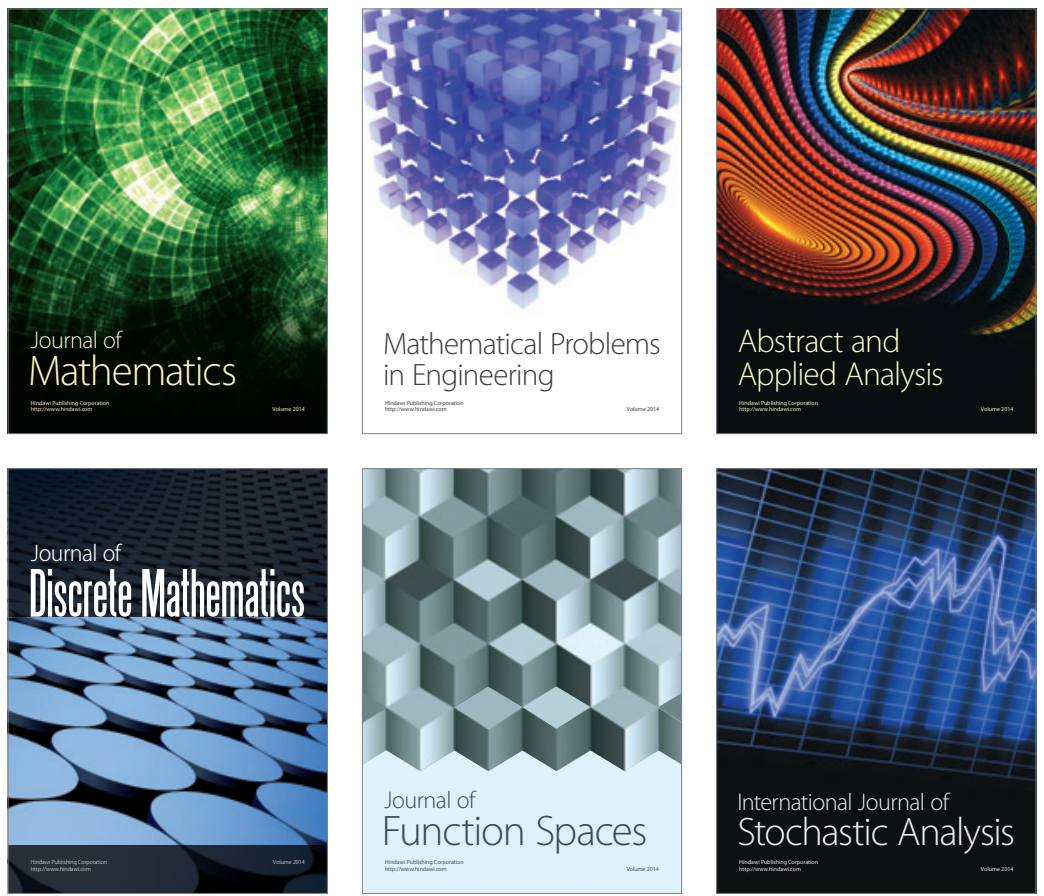

Journal of

Function Spaces

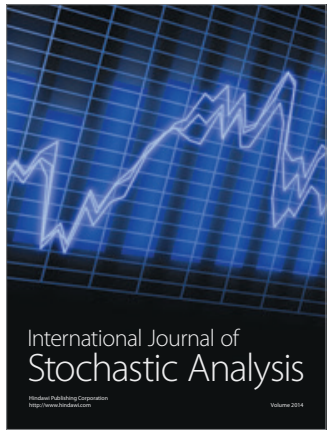

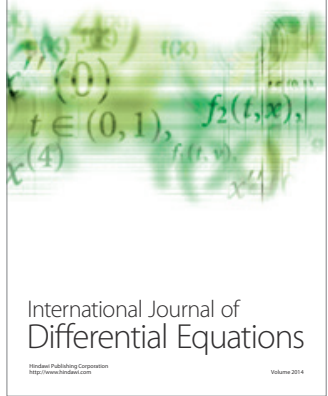
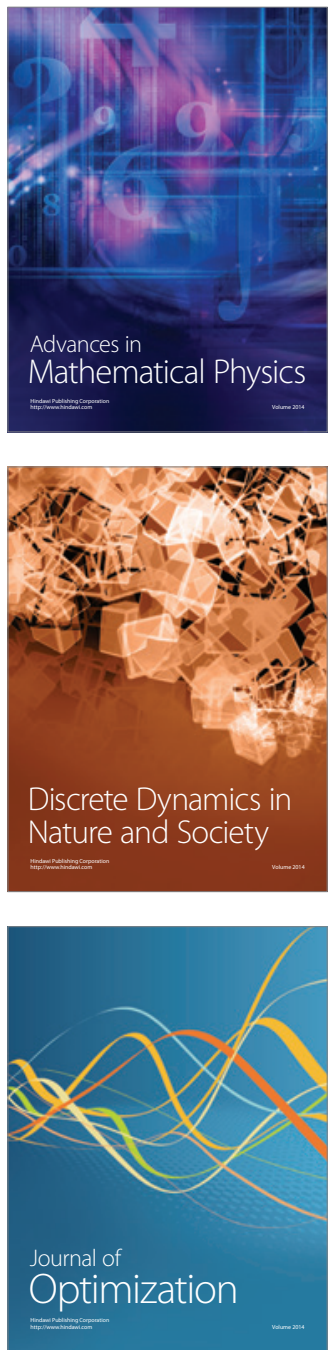